\title{
Resonant processes and Coulomb interactions in $\left(\mathrm{C}_{59} \mathrm{~N}\right)_{2}$
}

\author{
K. Schulte, L. Wang, ${ }^{\text {a) }}$ and P. J. Moriarty \\ School of Physics and Astronomy, University of Nottingham, Nottingham NG7 2RD, United Kingdom \\ K. Prassides \\ Department of Chemistry, University Science Laboratories, South Road, Durham DH1 3LE, \\ United Kingdom \\ N. Tagmatarchis \\ National Hellenic Research Foundation, Theoretical and Physical Chemistry Institute, \\ 48 Vasileos Constantinou Avenue, 11635 Athens, Greece
}

(Received 9 February 2007; accepted 22 March 2007; published online 11 May 2007)

\begin{abstract}
We have determined the on-site molecular Coulomb interaction energy $U$ of a $\left(\mathrm{C}_{59} \mathrm{~N}\right)_{2}$ bulk film and find values ranging from $1.10 \pm 0.10 \mathrm{eV}$ for the highest occupied molecular orbital to $1.35 \pm 0.10 \mathrm{eV}$ for the deeper lying orbitals, comparable to values found in $\mathrm{C}_{60}$. The on-site Coulomb interaction between a carbon core hole and valence electrons, $U_{c}$, is, however, substantially lower than in $\mathrm{C}_{60}$ at $1.35 \pm 0.07 \mathrm{eV}$. Resonant photoemission (RESPES) results show a weakened participator decay channel, especially around the $\mathrm{N} 1 s$ threshold, where resonance of the highest occupied molecular orbital shoulder is absent. Near-edge x-ray absorption fine structure and constant initial state measurements, taken in parallel with the RESPES data, indicate, however, that matrix element effects cannot be ruled out. (C) 2007 American Institute of Physics.
\end{abstract}

[DOI: $10.1063 / 1.2730787]$

\section{INTRODUCTION}

15 years ago combined photoemission (PES), inverse photoemission (IPES), and Auger measurements by Lof et al. placed the molecular solid $\mathrm{C}_{60}$ within the class of correlated electron systems. ${ }^{1}$ They found a relatively large ratio of $U$, the Coulomb interaction between electrons in the valence band, and the bandwidth $W$ of $U / W \sim 3-4$, comparable to the values for high $T_{c}$ cuprates. It is intriguing that this Hubbard $U$ terminology, regularly used for atomic, transition metal structures, applies so well to a large organic molecule. Even upon electron doping the picture continues to hold, as has been studied by Brühwiler et al. for three phases of $\mathrm{K}_{x} \mathrm{C}_{60}{ }^{2}$, where they found that $U$ approximately has a value of $1.5 \mathrm{eV}$ for all three compounds, but activation of intermolecular charge-transfer screening reduces the measured energy value in $\mathrm{K}_{3} \mathrm{C}_{60}$ to $0.6 \mathrm{eV}$.

One might ask how these fundamental processes and parameters are influenced by different forms of doping, such as in endohedral fullerenes or substitutionally doped $\mathrm{C}_{59} \mathrm{~N}$. With this in mind we have conducted a spectroscopic study of $\mathrm{C}_{59} \mathrm{~N}$, or azafullerene. In this molecule one carbon atom is replaced by a nitrogen atom producing, effectively, a form of $n$-type doping. This substitution affects the bonding of the carbon cage in such a way that the preferred structure at room temperature is a $\left(\mathrm{C}_{59} \mathrm{~N}\right)_{2}$ dimer, as depicted in Fig. 2, where an intermolecular $s p^{3}$-like bond between two carbons neighboring the nitrogen atoms is formed, with a predicted binding energy of the order of $0.65 \mathrm{eV} .^{3}$ The influence of this substitution is twofold: first, the stronger core potential of-

\footnotetext{
${ }^{a)}$ Present address: Physics Department, National University of Singapore, Singapore 117542, Singapore.
}

fered by the nitrogen atom manifests itself in a low binding energy shoulder split off from the highest occupied molecular orbital (HOMO), carrying significant nitrogen character. ${ }^{4}$ This is, however, not to be associated with simple filling of the lowest unoccupied molecular orbital (LUMO) of $\mathrm{C}_{60}$, as the excess electronic charge stays relatively localized on the nitrogen sites. Second, the dimerization itself also introduces changes to the electronic structure, observed as spectral broadening and the appearance of two extra structures in between the three highest valence band peaks in photoemission. ${ }^{5}$

As compared to $\mathrm{C}_{60}$, the gap between occupied and unoccupied states is smaller in $\left(\mathrm{C}_{59} \mathrm{~N}\right)_{2}{ }^{6}$ This is also confirmed by optical and electron energy loss experiments: $\left(\mathrm{C}_{59} \mathrm{~N}\right)_{2}$ shows a clear onset at $1.4-1.55 \mathrm{eV}$ and a further strong peak at $1.8 \mathrm{eV}$ which are both optically allowed, whereas in $\mathrm{C}_{60}$ the onset at $1.55 \mathrm{eV}$ is extremely weak and the nearest optically allowed transition does not occur until $2 \mathrm{eV}^{6-8}$ It is thus feasible to assume that the larger size, explicit coupling, and reduced symmetry of the molecule could offer extra delocalization and polarization possibilities for these optical excitons and other fundamental charge-transfer excitations. This should manifest itself in the molecular Coulomb interaction or the screening processes.

\section{EXPERIMENTAL DETAILS}

All experiments reported here were performed at the undulator beamline I311 at the MAX II storage ring in MAXlab, Lund, Sweden. ${ }^{10}$ The overall resolution of the spectra varied between 30 and $260 \mathrm{meV}$ for photon energies ranging from 33 to $600 \mathrm{eV}$. The spectra reported here are recorded at room temperature and at normal emission, with $55^{\circ}$ as the 
fixed angle between incoming beam and analyzer. For the near-edge x-ray absorption fine structure (NEXAFS) and the constant initial state (CIS) spectra, the Scienta analyzer was used with a pass energy of $50 \mathrm{eV}$ [electron energy window $($ eew $)=4.3 \mathrm{eV}$ ] for the $\mathrm{C} K$ edge and $200 \mathrm{eV}($ eew $=17 \mathrm{eV})$ for the $\mathrm{N} K$ edge. All spectra are normalized to the ring current and, where appropriate, corrected for the photon energy profile of the beamline by measurements on a clean $\mathrm{Ag}: \operatorname{Si}(111)-(\sqrt{3} \times \sqrt{3}) R 30^{\circ}$ surface, which was used as a substrate. Details of preparation of this surface can be found elsewhere. ${ }^{12}$ Cleanliness and quality of this surface reconstruction were checked by means of photoemission and low energy electron diffraction. A small residual carbon peak at $283.3 \mathrm{eV}$ was found. The intensity of this peak on the silver terminated surface was less than $0.5 \%$ compared to the intensity of the C $1 s$ peak of the final, thick film of $\left(\mathrm{C}_{59} \mathrm{~N}\right)_{2}$ and therefore does not hamper the quality or interpretation of the reported data. Energy calibration of the photon energy was achieved by measuring strong photoemission peaks in first and second order lights, without changing the parameters for the beamline or spectrometer. The binding energy of the photoemission and NEXAFS peaks was determined (1) relative to the Fermi edge of a gold foil or tantalum sample holder clip in electrical contact with the sample and/or (2) relative to the vacuum by means of applying bias voltages of 7 and $10 \mathrm{~V}$ to the sample, following the method of Schnadt et al., ${ }^{11}$ which also allows for direct comparison to similar measurements taken on $\mathrm{C}_{60} \cdot{ }^{13}$

The azafullerene molecules used in this study were prepared and purified as described elsewhere ${ }^{14}$ and deposited from a standard Ta-crucible Knudsen cell after baking and prolonged degassing at $200-250{ }^{\circ} \mathrm{C}$. The $\left(\mathrm{C}_{59} \mathrm{~N}\right)_{2}$ film was deposited at $\sim 400-450{ }^{\circ} \mathrm{C}$ in three sequential bouts to ensure a homogeneous buildup of the thick film. The first two deposits of $30 \mathrm{~min}$ were made with the sample kept at $350{ }^{\circ} \mathrm{C}$ (first) and $300{ }^{\circ} \mathrm{C}$ (second) to aid homogeneous layer-by-layer growth. Although $\mathrm{C}_{59} \mathrm{~N}$ sublimes in monomer form, dimer formation is recovered in multilayers. ${ }^{15}$ The dimers arrange in a monoclinic unit cell, similar to that of $\mathrm{Rb}_{1} \mathrm{C}_{60} \cdot{ }^{16,17}$ After the second deposition the $\mathrm{C} 1 s$, Si $2 p$, and valence band signals showed that coverage close to a monolayer was established, although exact calibration is difficult as a certain amount of island formation cannot be ruled out. Using our warm deposited near monolayer as a growth template the final, $1 \mathrm{~h}$ long deposition was carried out on an unheated substrate in order to obtain a bulklike film. This was indeed achieved as no silicon-or silver-related signal was visible up to photon energies of $500 \mathrm{eV}$.

Thick (semiconducting) films, however, can be prone to charging during spectroscopy experiments. In order to test this we took a number of consecutive, quick measurements of the $\mathrm{C} 1 s$ peak and looked for any signs of peak shifting and/or broadening. We also applied several bias voltages to the sample in the range from 4 to $10 \mathrm{~V}$ and observed whether the shift was carried through homogeneously in the whole spectrum (and therefore also at different depths in the film) from cutoff to the $\mathrm{C} 1 \mathrm{~s}$ peak at a photon energy of $350 \mathrm{eV}$. No signs of charging were observed.

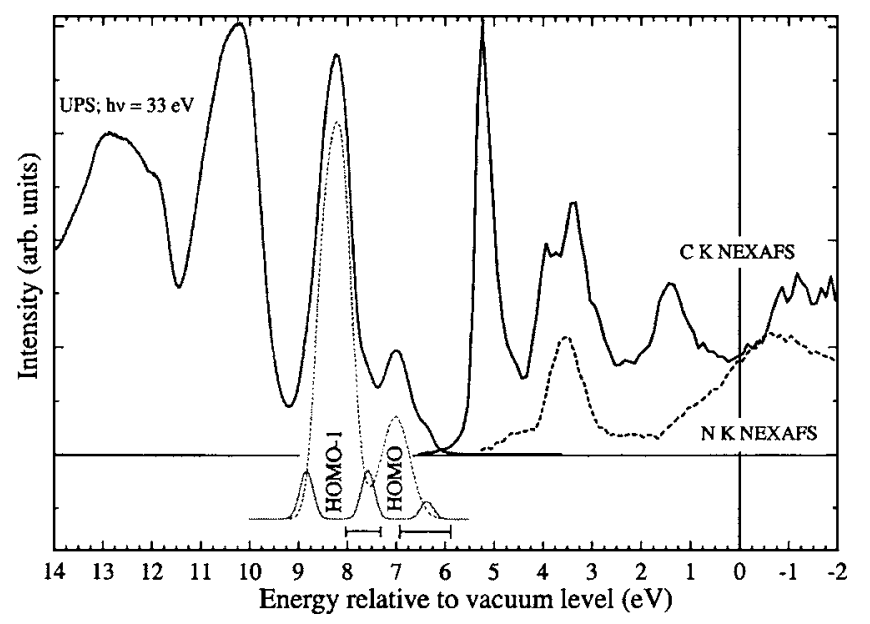

FIG. 1. Combined valence band PES, C $K$, and $\mathrm{N} K$ NEXAFS spectra plotted on a common energy scale, referenced to the vacuum level (Ref. 18). The Gaussian peaks underneath the PES spectrum indicate the HOMO and HOMO-1 (gray, dashed), which are similar to those of $\mathrm{C}_{60}$. The other three peaks at $6.37\left(\mathrm{HOMO}^{+S}\right), 7.58\left(\mathrm{HOMO}-1^{+S}\right)$, and $8.84 \mathrm{eV}\left(\mathrm{HOMO}-1^{-S}\right)$ indicate peaks with appreciable nitrogen character (gray, solid). The horizontal bars indicate the energy windows used in the constant initial state measurements (see text).

\section{RESULTS AND DISCUSSION}

Figure 1 shows the characteristic valence band of $\left(\mathrm{C}_{59} \mathrm{~N}\right)_{2}$ referenced to the vacuum level. The influence of the nitrogen doping is evidenced most clearly in the $\hbar \omega$ $=33 \mathrm{eV}$ spectrum by the low binding energy $(\mathrm{BE})$ shoulder on the $7 \mathrm{eV}$ peak. However, we will refer to this shoulder as $\mathrm{HOMO}^{+s}$, reserving the titles HOMO and HOMO-1 for the peaks at 7 and $8.3 \mathrm{eV}$, respectively. This is to facilitate direct comparison with $\mathrm{C}_{60}$.

Other signatures of nitrogen doping and dimer formation are the shoulder on the low BE side of the HOMO-1, which we will denote by HOMO- $1^{+s}$, and the shoulder on the high $\mathrm{BE}$ side of the HOMO-1 peak, which we will denote by HOMO $-1^{-s}$. All three peaks are reported to have appreciable nitrogen character. ${ }^{4}$

On the same energy scale we have also plotted the carbon $K$ edge and nitrogen $K$ edge NEXAFS spectra, which are a measure of the partial carbon and nitrogen densities of unoccupied states, respectively. ${ }^{18}$ The $\mathrm{C} K$ NEXAFS spectrum is again very similar to that of $\mathrm{C}_{60}$ (see, for example, Ref. 4). We have shifted the nitrogen spectra to lower energies by $115.52 \mathrm{eV}$, which is the difference between the $\mathrm{N} 1 \mathrm{~s}$ and $\mathrm{C} 1 \mathrm{~s}$ core level binding energies $(405.22-289.70 \mathrm{eV})$ with respect to the vacuum level. This shows that the LUMO of $\left(\mathrm{C}_{59} \mathrm{~N}\right)_{2}$ has predominantly carbon character. The gap between the $\left(\mathrm{C}_{59} \mathrm{~N}\right)_{2}$ HOMO and LUMO in this plot is $\sim 1.1 \mathrm{eV}$ peak to peak. This, however, cannot be directly associated with the transport gap observed in combined PESIPES as the presence of a core hole in the NEXAFS spectra will shift the unoccupied spectra to lower binding energies. Rather, the observed gap $\Delta E_{\mathrm{XAS}}$ in NEXAFS should be of the order of $\Delta+U-U_{c}$, where $\Delta$ is the true HOMO-LUMO splitting, $U$ is the on-site molecular Coulomb interaction, and $U_{c}$ is the on-site Coulomb interaction between the core hole and valence electrons. ${ }^{13}$ From EELS and optical absorption 


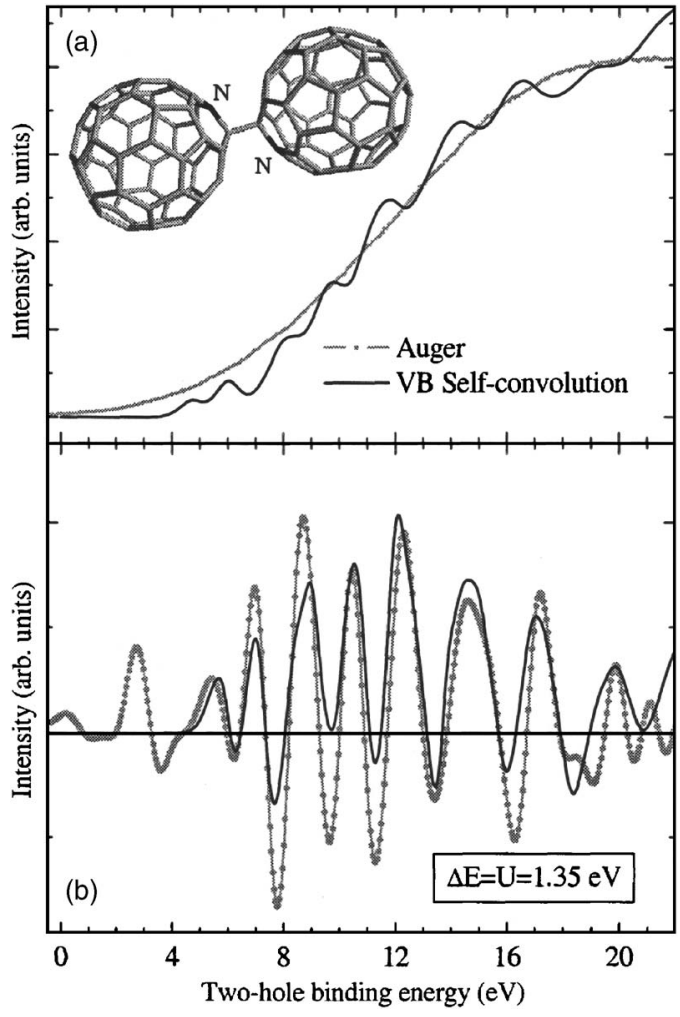

FIG. 2. (a) Auger and self-convoluted VB data on two-hole binding energy scale, inset: molecular structure of $\left(\mathrm{C}_{59} \mathrm{~N}\right)_{2}$. (b) Bandpass filtered data, showing the enlarged wiggles. A $1.35 \mathrm{eV}$ rigid shift gives a good overall alignment of the self-convoluted valence band with the Auger data. The HOMO peak, however, is better served by a $1.1 \mathrm{eV}$ shift.

measurements we have $\Delta=1.4 \mathrm{eV} .^{7,8}$ In order to extract the on-site molecular Coulomb interaction $U$ we follow the procedure used by Lof et al. ${ }^{1}$ We first self-convolved the valence band spectrum, referenced to the Fermi level measured on a gold foil in contact with the sample. This convolution mimics a state which has two uncorrelated holes in the valence band. We then compare this to the Auger spectrum, which is a true two-hole per molecule state, and therefore will carry information on the extra Coulomb interaction between the holes within it. Figure 2(a) depicts the $\mathrm{C} 1 s \mathrm{KVV}$ Auger spectrum of $\left(\mathrm{C}_{59} \mathrm{~N}\right)_{2}$ taken at a photon energy $E_{\mathrm{ph}}$ $=500 \mathrm{eV}$. The kinetic energy scale is converted to a two-hole binding energy scale by subtracting it from the binding energy of the $\mathrm{C} 1 s$ peak relative to the Fermi level of the Au foil $(285.07 \pm 0.08 \mathrm{eV})$.

In order to accentuate the "wiggles" of the spectrum more clearly, we have used a bandpass filter, thereby eliminating the noise as well as the overall smooth shape. We can then compare the two spectra, as plotted in Fig. 2(b). In order to obtain the best overall overlap of the structures we have to shift the self-convolution curve rigidly by $1.35 \mathrm{eV}$, or $U$ in other words, which is only slightly lower than the values obtained for $\mathrm{C}_{60}$ [1.4-1.6 eV (Refs. 1 and 2)]. This shift holds for all but the HOMO: the lowest binding energy feature in the self-convoluted spectrum is better served by a smaller shift of $U=1.1 \mathrm{eV}$ [1.2 eV in $\mathrm{C}_{60}$ (Ref. 19)].

The additional peak at $\sim 3 \mathrm{eV}$ in the Auger spectrum can be attributed to a screening decay channel involving electron transfer from a neighboring molecule before Auger decay occurs, which cannot be reproduced in the valence band (VB) self-convolution. Even though this peak is also observed in the Auger spectra of $\mathrm{C}_{60}$ (Ref. 19) and to a lesser extent in $\mathrm{K}_{6} \mathrm{C}_{60}$, ${ }^{2}$ it is far more pronounced in $\left(\mathrm{C}_{59} \mathrm{~N}\right)_{2}$, indicating a heightened probability for charge transfer in the presence of a carbon core hole in this compound. We believe that this increase is a direct consequence of the excess nitrogen charge and explicit connection between two fullerene bulbs, giving rise to an enhanced polarization per molecule. The polarizability $\alpha$ has been reported as $225 \AA^{3}$ in calculations, ${ }^{20}$ and can also be derived from the value of the dielectric constant at zero frequency, $\varepsilon(0)=5.6,{ }^{21}$ through the Clausius-Mosotti relation, giving $\alpha=240 \AA^{3}$ or via the method of Miller and Savchik, ${ }^{22}$ yielding $\alpha=253 \AA^{3}$ (we will use the mean value of $240 \AA^{3}$ ). This is indeed more than a double enhancement of the value for $\mathrm{C}_{60}$, where $\alpha$ $=76.5-90 \AA^{3}{ }^{21,23}$

We can now also determine a value for $U_{c}$ using $\Delta+U$ $-\Delta E_{\mathrm{XAS}}=1.4+1.1-1.1=1.4 \pm 0.1 \mathrm{eV}$. This is somewhat lower than in $\mathrm{C}_{60}$, although reported values differ strongly $\left[U_{c}=1.54 \mathrm{eV},{ }^{13} 1.8 \mathrm{eV},{ }^{9}\right.$ and $2.2 \mathrm{eV}$ (Ref. 24)]. The high value of $2.2 \mathrm{eV}$ was determined in an alternative way, which we will also employ here to obtain $U_{c}$ in order to check our finding. Brühwiler et al. determined $U_{c}$ from $U_{c} \simeq \mathrm{C}^{\mathrm{XPS}} 1 \mathrm{~s}$ $-\mathrm{C}^{\mathrm{LUMO}} 1 s-\mathrm{EA}-E_{\mathrm{pol}}$ by taking the difference between the C $1 s$ binding energy measured in XPS (referenced to the vacuum) and the excitation energy of $\mathrm{C} 1 s$ to LUMO in NEXAFS, and then subtracting the electron affinity (EA) corrected for the polarization screening in the solid $\left(E_{\mathrm{pol}}\right)$. Theoretical results give $\mathrm{EA}=2.85 \mathrm{eV}{ }^{3}$ The polarization energy can be readily calculated from

$$
E_{\mathrm{pol}}=\frac{n \alpha e^{2}}{2\left(4 \pi \varepsilon_{0}\right) R^{4}}
$$

with $n$ the number of nearest neighbors, $\alpha$ the polarizability, and $R$ the nearest neighbor distance. ${ }^{25}$ The number of nearest neighbors in the monoclinic $\left(\mathrm{C}_{59} \mathrm{~N}\right)_{2}$ crystal that lie side to side with the central $\left(\mathrm{C}_{59} \mathrm{~N}\right)_{2}$ molecule under consideration is 6 , and are located at an average distance of $9.97 \AA{ }^{16}$ giving $E_{\mathrm{pol}}=1.05 \mathrm{eV}$ [0.7-0.8 eV in $\mathrm{C}_{60}$ (Refs. 1 and 26)]. We then obtain $U_{c} \simeq 289.70-284.47-2.85-1.05=1.3 \pm 0.1 \mathrm{eV}$ which is in excellent agreement with our previous finding. Taking these results together, we confirm a relative average reduction of around $0.5 \mathrm{eV}$ for $U_{c}$ in $\left(\mathrm{C}_{59} \mathrm{~N}\right)_{2}$ as compared to $\mathrm{C}_{60}$.

Figure 3(a) shows the valence band spectrum at $h \nu$ $=284.6 \mathrm{eV}$, which resonantly excites a $\mathrm{C} 1 \mathrm{~s}$ electron into the LUMO, together with two valence band spectra taken at offresonance energies below this threshold, and an Auger background. The two off-resonance spectra allow an elimination of the second order C $1 s$ signal which "travels through" the valence band as indicated.

In Fig. 3(b) the same is presented for resonant $\mathrm{N} 1 s$ photoemission. Here we have added, however, a further four spectra taken at photon energies around the maximum resonance (precise photon energies are indicated by vertical lines in Fig. 4-color online). These were averaged together with 


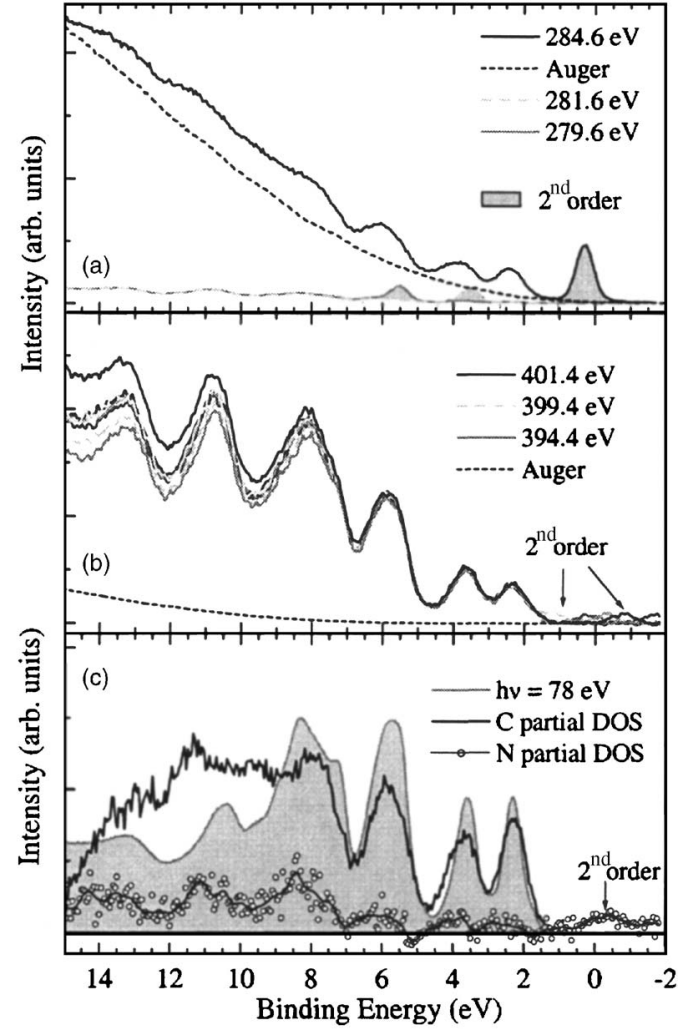

FIG. 3. (a) C $1 s$ RESPES, showing the on-resonant and two off-resonant spectra, plus Auger background, (b) N 1s RESPES, showing max-resonant, Auger, and two off-resonant spectra, the other spectra were taken at energies around the maximum resonance, as indicated in Fig. 4(b) (color online), (c) Partial DOS obtained by subtracting the Auger curve plus an average of off-resonant spectra from on-resonant spectrum. For the N RESPES data, a combination of the "on-resonant" spectra was used to minimize noise.

the maximum resonance spectra to achieve lower noise levels, as the resonant effects of the nitrogen density of states are very weak.

When analyzing the resonant photoemission (RESPES) spectra, we have to consider three relaxation processes. After the initial process of exciting a core electron into an unoccupied orbital, the decay of this highly energetic state will always involve refilling the core hole, but the final state of the molecule is characterized by (a) the resonant or participator process, which leaves the system behind with one hole in the valence band ( $\mathrm{N}-1$ electron state). This is equivalent to the final state in normal valence band photoemission. Both normal and resonantly excited processes occur simultaneously and will interfere to yield an enhancement of the element's contribution to the valence band; (b) the spectator process, in which the initially excited core electron still resides in the unoccupied orbital and the valence band carries two holes. Although this is again a $\mathrm{N}-1$ electron final state, the molecule is in an excited state and contributions from this process to the photoemission spectrum will therefore be separated in energy from the participator part; (c) Auger decay: the excited core electron may leave the atom (or molecule) leaving a valence band electron to annihilate the core hole, while another electron is ejected. The system is thus left behind in a $\mathrm{N}-2$ final state, and this contribution is again separated from the participator contribution to the spectrum,
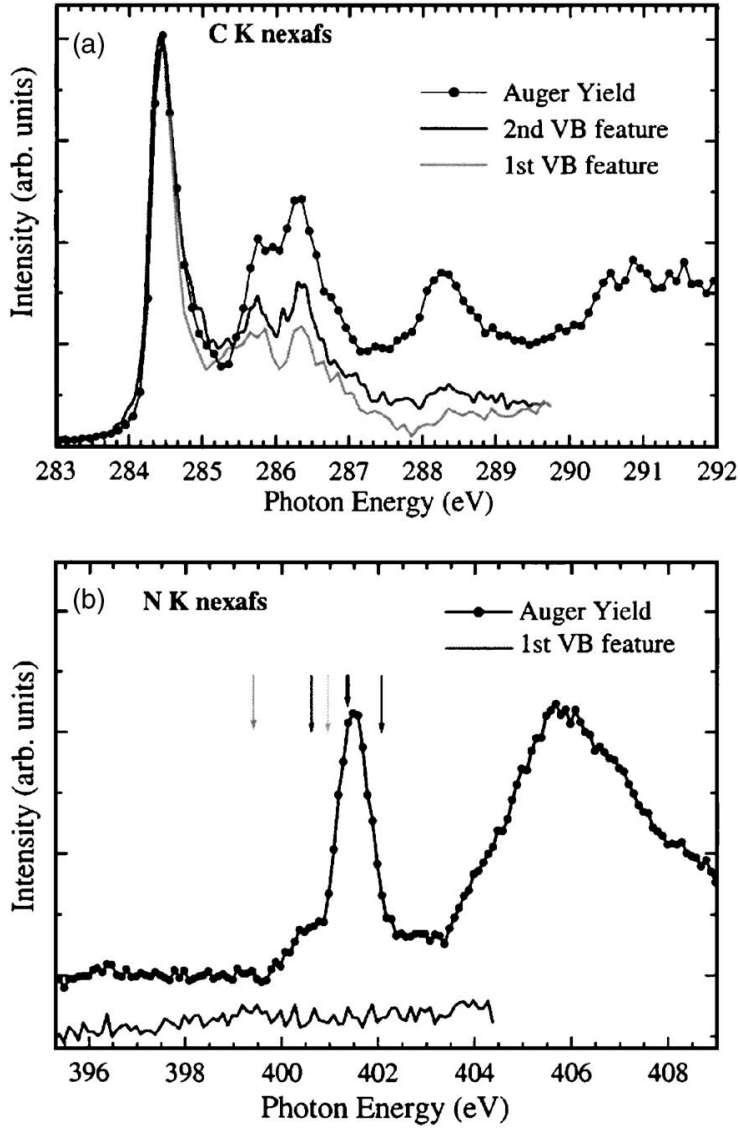

FIG. 4. (a) $\mathrm{C} K$ NEXAFS (Auger yield) and CIS data-BE regions indicated in Fig. 1 by horizontal bars, (b) $\mathrm{N} K$ NEXAFS and CIS data (BE region: see Fig. 1 and text), the CIS curve is multiplied by a factor of 9 and shifted down for clarity.

although overlap with the spectator part can be less straightforward to determine.

In Fig. 3(c) we have plotted the resonant valence band spectra after subtracting the Auger and nonresonant part, which should therefore give a measure of the partial $\mathrm{N}$ and $\mathrm{C}$ densities of states (DOSs), predominantly correct at low binding energies (as we cannot adequately correct for the spectator contribution). Even though the $\mathrm{N}$ partial DOS spectrum is still relatively noisy due to low intensity, there is a slight resonance visible at the HOMO- $1^{+S}$ and HOMO- $1^{-S}$ positions, along with more enhanced deeper binding energy peaks. This is qualitatively consistent with theoretical calculations, although the HOMO- $1^{ \pm S}$ parts could have been expected to show higher intensity. ${ }^{4}$ The surprising thing is that the $\mathrm{HOMO}^{+S}$, which should also contain significant nitrogen character, does not resonate at all.

Brühwiler et al. have conducted in depth studies of the decay processes in matrix-isolated $\mathrm{C}_{60}$ and thin films of $\mathrm{C}_{60}$ compared to the bulk, ${ }^{9,24}$ and found that there is evidence for localization of varying degrees for all absorption transitions possible below the ionization threshold. This can be ascertained by a strong manifestation of participator decay, measurable in both RESPES and indirectly via CIS spectra. We can then compare these measurements to total yield (or partial or Auger yield) measurements, which are a better representation of the unoccupied state behavior. We have per- 
formed a CIS measurement (BE region indicated by horizontal bar in Fig. 1) on the $\mathrm{HOMO}^{+S}$ peak through the nitrogen $K$ edge region in order to confirm that any resonance is truly absent. This is plotted in Fig. 4(b), alongside Auger yield spectra, and indeed confirms that the low binding energy shoulder does not resonate.

This can be explained either in terms of an intramolecular matrix effect-if the overlap of an occupied orbital with that of the excited $\mathrm{N} 1 s$ core electron is weak or even symmetry forbidden, the resonant process will be strongly reduced-or in terms of delocalization. In this second picture the excited electron might transfer from the nitrogen LUMO into unoccupied carbon-dominated states (see Fig. 1) or even transfer to a different molecule, leaving the nitrogen atom to relax via Auger decay. The higher mobility of this "extra" electron has also been observed in $\mathrm{C}_{59} \mathrm{~N}-\mathrm{C}_{60}$ (Ref. 27) and forms the more plausible explanation. Indeed, if we tentatively compare our nitrogen LUMO position (on a core hole excited molecule) to inverse photoemission data of $\mathrm{C}_{60}$, referenced to the vacuum level ${ }^{9}$ (representing the unoccupied carbon density of states of the background material), an excellent overlap with the carbon LUMO centered at $3.4 \mathrm{eV}$ is achieved.

In order to complete the picture, we have also examined resonance processes around the carbon $K$ edge. In Fig. 3(a) it is clear that for the three lowest binding energy features a significant degree of participator contribution can be inferred as they clearly stand out against the more formless background due to Auger and spectator decay. Comparing the resonance spectrum of $\left(\mathrm{C}_{59} \mathrm{~N}\right)_{2}$ to those of $\mathrm{C}_{60}$ reported in literature $^{9,28}$ we do, however, observe a slight reduction in resonant enhancement at the LUMO excitation energy $(284.5 \mathrm{eV})$. This is strongest for the HOMO (factor of 1.3) and less so for HOMO-1 (factor of 1.2) and HOMO-2 (factor of 1.1) peak. The Auger background strength and peak widths are, however, similar. We have again carried out CIS measurements, now on two separate regions in the valence band (again indicated by horizontal lines in Fig. 1), and compared these to Auger yield NEXAFS. The results are presented in Fig. 4(a). All curves are normalized to the LUMO intensity. Comparing our findings to those of Brühwiler et al. ${ }^{28}$ we observe a relative reduction in the weight of the LUMO compared to that of the higher unoccupied states. Also, in contrast to $\mathrm{C}_{60}$, the $\mathrm{LUMO}+1$ intensity is relatively enhanced for both valence band regions with respect to the Auger yield spectrum. Both these observations confirm a shift of resonant intensity in the valence band away from the LUMO excitation towards higher unoccupied states, which is hard to explain in terms of an increased mobility of the excited core electron. An explanation for the carbon core hole decay therefore needs to be sought in terms of matrix overlap that has resulted in a stronger coupling between occupied orbitals and the carbon $\mathrm{LUMO}+1$ and $\mathrm{LUMO}+2$, as opposed to the LUMO coupling. For the nitrogen electrons, however, the low resonance intensity in the valence band, together with the absence of any resonance for the $\mathrm{HOMO}^{+S}$, means that delocalization is still a probable explanation.

\section{CONCLUSIONS}

In this paper we have presented electron spectroscopy measurements for $\left(\mathrm{C}_{59} \mathrm{~N}\right)_{2}$ and deduced the value of the molecular on-site Coulomb interaction $U$ to be $1.35 \pm 0.10 \mathrm{eV}$ ( $1.1 \mathrm{eV}$ for the HOMO). We have also determined the on-site carbon core hole-valence band electron interaction and found an average value of $1.35 \pm 0.07 \mathrm{eV}$. Both values are lower than those for $\mathrm{C}_{60}$ and would be consistent with reduced participator intensity in C $1 s$ RESPES. In the C $K$ CIS scans of the first and second valence band peaks we find, however, that the intensity of especially the carbon LUMO+1 peak is increased relative to that of the LUMO, which is more consistent with an explanation in terms of matrix element overlap between excited electron states and occupied orbitals. In the nitrogen RESPES and CIS measurements we find no resonance for the $\mathrm{HOMO}^{+S}$ peak, which in principle should carry significant nitrogen character and only minimal enhancement of the other low binding energy regions where nitrogen partial DOS should be appreciable. Here the effects of matrix elements more strongly compete with the possibility of delocalizing the excited electron due to overlap in energy between unoccupied nitrogen-dominated levels and unoccupied carbon states, which agrees with a picture of a nitrogen impurity in a larger molecule. To fully elucidate these resonance processes, theoretical efforts are needed.

\section{ACKNOWLEDGMENTS}

The authors gratefully acknowledge support by the European Community-Research Infrastructure Action under the FP6 "Structuring the European Research Area" Programme (through the Integrated Infrastructure Initiative "Integrating Activity on Synchrotron and Free Electron Laser Science") and express their thanks to Jonas Weissenrieder and the staff at MAXlab. One of the authors (K.S.) acknowledges the Nanospectra network under Grant No. HPRN-CT2002-00320 and the EPSRC for funding under Grant No. GR/01880/01. This work, conducted as part of the award ("Functionalization of Carbon Nanotubes Encapsulating Novel Carbon-based Nanostructured Materials") made under the European Heads of Research Councils and Curopean Science Foundation EURYI (European Young Investigator) Awards scheme, was supported by funds from the Participating Organizations of EURYI and the EC Sixth Framework Programme.

\footnotetext{
${ }^{1}$ R. W. Lof, M. A. van Veenendaal, B. Koopmans, H. T. Jonkman, and G. A. Sawatzky, Phys. Rev. Lett. 68, 3924 (1992).

${ }^{2}$ P. A. Brühwiler, A. J. Maxwell, A. Nilsson, N. Mårtensson, and O. Gunnarsson, Phys. Rev. B 48, 18296 (1993).

${ }^{3}$ W. Andreoni, Annu. Rev. Phys. Chem. 49, 405 (1998).

${ }^{4}$ T. Pichler, M. Knupfer, M. S. Golden et al., Phys. Rev. Lett. 78, 4249 (1997).

${ }^{5}$ B. S. Itchkawitz, J. P. Long, T. Schedel-Niedrig, M. N. Kabler, A. M. Bradshaw, R. Schlögl, and W. R. Hunter, Chem. Phys. Lett. 243, 211 (1995).

${ }^{6}$ S. Haffner, T. Pichler, M. Knupfer et al., Eur. Phys. J. B 1, 11 (1998).

${ }^{7}$ W. Plank, T. Pichler, H. Kuzmany, O. Dubay, N. Tagmatarchis, and K. Prassides, Eur. Phys. J. B 17, 33 (2000).

${ }^{8}$ C. Silien, I. Marenne, J. Auerhammer, N. Tagmatarchis, K. Prassides, P. A. Thiry, and P. Rudolf, Surf. Sci. 482-485, 1 (2001).
} 
${ }^{9}$ P. A. Brühwiler, O. Karis, and N. Mårtensson, Rev. Mod. Phys. 74, 703 (2002).

${ }^{10}$ R. Nyholm, J. N. Andersen, U. Johansson, B. N. Jensen, and I. Lindau, Nucl. Instrum. Methods Phys. Res. A 467-468, 520 (2001).

${ }^{11}$ J. Schnadt, J. N. O’Shea, L. Patthey, J. Krempaský, N. Mårtensson, and P. A. Brühwiler, Phys. Rev. B 67, 235420 (2003).

${ }^{12}$ L. Wang, K. Schulte, R. A. J. Woolley et al., Surf. Sci. 564, 156 (2004).

${ }^{13}$ R. Schwedhelm, L. Kipp, A. Dallmeyer, and M. Skibowski, Phys. Rev. B 58, 13176 (1998).

${ }^{14}$ J.-C. Hummelen, B. Knight, J. Pavlovich, R. Gonzalez, and F. Wudl, Science 269, 1554 (1995).

${ }^{15}$ M. J. Butcher, F. H. Jones, P. H. Beton et al., Phys. Rev. Lett. 83, 3478 (1999).

${ }^{16}$ C. M. Brown, L. Cristofolini, K. Kordatos et al., Chem. Mater. 8, 2548 (1996).

${ }^{17}$ T. Guo, C. Jin, and R. E. Smalley, J. Phys. Chem. B 95, 4948 (1991).

${ }^{18}$ Effectively, down shifting the $\mathrm{N} K$ edge spectrum merely places it on the same photon energy scale as the C $K$ edge spectra and not on the used absolute energy scale together with the valence band. However, since it demonstrates the relative positioning of the unoccupied orbital character, we have chosen to include it in this way.

${ }^{19}$ R. W. Lof, M. A. van Veenendaal, H. T. Jonkman, and G. A. Sawatzky, J. Electron Spectrosc. Relat. Phenom. 72, 83 (1995).

${ }^{20}$ J. Dong, J. Jiang, J. Yu, Z. D. Wang, and D. Y. Xing, Phys. Rev. B 52, 9066 (1995)

${ }^{21}$ T. Pichler, M. Knupfer, R. Friedlein et al., Synth. Met. 86, 2313 (1997).

${ }^{22}$ K. J. Miller and J. Savchik, J. Am. Chem. Soc. 101, 7206 (1979).

${ }^{23}$ R. Antoine, P. Dugourd, D. Rayane, E. Benichou, M. Broyer, F. Chandezon, and C. Guet, J. Chem. Phys. 110, 9771 (1999).

${ }^{24}$ P. A. Brühwiler, A. J. Maxwell, P. Rudolf, C. D. Gutleben, B. Wästberg, and N. Mårtensson, Phys. Rev. Lett. 71, 3721 (1993).

${ }^{25}$ D. K. G. de Boer, C. Haas, and G. A. Sawatzky, Phys. Rev. B 29, 4401 (1984).

${ }^{26}$ R. Hesper, L. H. Tjeng, and G. A. Sawatzky, Europhys. Lett. 40, 177 (1997).

${ }^{27}$ A. Rockenbauer, G. Cśanyi, F. Fülöp, S. Garaj, L. Korecz, R. Lukács, L. Forró, S. Pekker, and A. Jánossy, Phys. Rev. Lett. 94, 066603 (2005).

${ }^{28}$ P. A. Brühwiler, A. J. Maxwell, A. Nilsson, R. L. Whetten, and N. Mårtensson, Chem. Phys. Lett. 193, 311 (1992). 